\title{
Role and importance of the expression of transcription factor FOXC2 in cervical cancer
}

\author{
JING WANG $^{1}$ and XIUJUAN YUE ${ }^{2}$ \\ Departments of ${ }^{1}$ Obstetrics and Gynecology and ${ }^{2}$ Obstetrics, Linyi Hospital of Traditional Chinese Medicine, \\ Linyi, Shandong 276000, P.R. China
}

Received March 17, 2017; Accepted June 8, 2017

DOI: 10.3892/ol.2017.7004

\begin{abstract}
The aim of the present study was to investigate the relationship between the expression of transcription factor forkhead box C2 (FOXC2) and the clinical features of cervical cancer. A total of 66 patients with cervical cancer, 42 patients with cervical intraepithelial neoplasia (CIN) and 25 patients with cervical inflammation were enrolled. The positive expression rates and expression levels of mRNA of FOXC2, E-cadherin, N-cadherin, vascular endothelial growth factor (VEGF), stromal cell-derived factor-1 (SDF-1), Notch protein and lymphatic vessel endothelial hyaluronan receptor-1 (LYVE-1) in cervical tissues were detected using immunohistochemistry and RT-PCR. The positive expression rates and expression levels of mRNA of FOXC2, N-cadherin, VEGF, SDF-1, Notch and LYVE-1 in cervical cancer were significantly higher than those in CIN, and those in the inflammatory tissues were the lowest, while the positive expression rate of E-cadherin in cervical cancer was lower than that in CIN, and that in the inflammatory tissues was the highest $(\mathrm{P}<0.05)$. The positive expression rates of FOXC2, N-cadherin, VEGF, SDF-1, Notch and LYVE-1 in patients with cervical cancer [human papillomavirus (HPV) positive, squamous cell carcinoma, Stages III-IV, maximal diameter $\geq 3.8 \mathrm{~cm}$ and low differentiation] were increased, and the positive expression rate of E-cadherin was decreased $(\mathrm{P}<0.05)$. Correlation analysis revealed that FOXC2 was positively correlated with the positive expression rates of $\mathrm{N}$-cadherin, VEGF, SDF-1, Notch and LYVE-1, and negatively correlated with $\mathrm{E}$-cadherin $(\mathrm{P}<0.05)$. In conclusion, the high expression of FOXC2 is correlated with the HPV infection, pathological pattern, clinical stage, tumor diameter and differentiation grade of cervical cancer, which may be involved in
\end{abstract}

Correspondence to: Dr Xiujuan Yue, Department of Obstetrics, Linyi Hospital of Traditional Chinese Medicine, 211 Jiefang Road, Lanshan, Linyi, Shandong 276000, P.R. China

E-mail: opkqsa42@163.com

Key words: forkhead box $\mathrm{C} 2$, cervical cancer, Notch signaling pathway, lymphangiogenesis the epithelial-mesenchymal transition, vascular and matrix formation, Notch signaling pathway and lymphangiogenesis.

\section{Introduction}

Cervical cancer is the most common malignant tumor of the female genital tract, and its incidence shows an increasing and younger trend annualy (1). Its occurrence is closely related to the human papillomavirus (HPV) infection and abnormal gene expression (2). Previous findings showed that cervical cancer results from the joint effects of multi-stage, multi-channel and multi-molecular factors (3).

Embryonic development-related genes, such as vascular endothelial growth factor (VEGF)-C, Wnt and forkhead box protein $\mathrm{C} 2$ (FOXC2) play important roles in the occurrence and development of cervical cancer $(4,5)$. The expression of FOXC2 can be abnormally high in various malignant tumors, including lung, breast, cervical, esophageal and colorectal cancer, which is closely related to the clinical characteristics, treatment effects and survival prognosis of tumors (6-8). FOXC2 is an important transcriptional regulatory factor in early embryonic stage, which is closely related to the lymphatic and vascular formation, structural differentiation and functional remodeling (9).

The aim of the present study was to investigate the relationship between the expression of FOXC2 and clinical features of cervical cancer, and whether it was related to epithelial-mesenchymal transition (EMT), vascular and matrix formation, Notch signaling pathway and lymphangiogenesis, to provide new targets for improving the early diagnosis, clinical intervention and prognostic evaluation of the disease.

\section{Patients and methods}

Patient data. A total of 66 patients with cervical cancer, 42 patients with cervical intraepithelial neoplasia (CIN) and 25 patients with cervical inflammation admitted to Linyi hospital from June 2015 to October 2016 were continuously selected, and informed consent was obtained. For patients with cervical cancer, the average age was $62.3 \pm 13.5$ years, and there were 35 cases (53.0\%) of positive HPV infection, 57 cases of squamous cell carcinoma, 9 cases of adenocarcinoma, 5 cases of Stage I, 25 cases of Stage II, 28 cases of Stage III and 8 cases of Stage IV. The average maximum tumor diameter 
Table I. Target gene sequences.

\begin{tabular}{llc}
\hline Target gene & \multicolumn{1}{c}{ Primer sequence } & Length (bp) \\
\hline FOXC2 & 5'-AGTCTCATGCAGGTTCGACCAGC-3' & 358 \\
& 5'-ACTAAGAGGGTCCCGGACTGGC-3' & \\
E-cadherin & 5'-ATCAAAGGTATCACGGCAAACG-3' & 479 \\
& 5'-CGGAGAGCTCGTCCACGTAT-3' & 337 \\
N-cadherin & 5'-GTGCCATTAGCCAAGGGAATTCAGC-3' & \\
VEGF & 5'-GCGTTCCTGTTCCACTCATAGGAG-3' & 332 \\
SDF-1 & 5'-ACTACTTCTCCCGCCGCTAC-3' & 269 \\
& 5'-GAAATCAAACAGAGGCCGCATG-3' & \\
Notch & 5'-GGTTTCATCCAGGATCGAGCAGG-3' & 446 \\
LYVE-1 & 5'-ACAAAGATGGTCACGGTCTGCC-3' & \\
GAPDH & 5'-ACTACTTCTCCCGCCGCTAC-3' & 179 \\
& 5'-GAAATCAAACAGAGGCCGCATG-3' & 225 \\
\hline
\end{tabular}

FOXC2, forkhead box C2; VEGF, vascular endothelial growth factor; SDF-1, stromal cell-derived factor-1; LYVE-1, lymphatic vessel endothelial hyaluronan receptor- 1 .

was $3.8 \pm 1.5 \mathrm{~cm}$, and there were 15 cases of low differentiation, 44 cases of moderate differentiation and 7 cases of high differentiation. For patients with CIN, the average age was $59.8 \pm 15.6$ years and there were 17 cases $(40.5 \%)$ of positive HPV infection, 15 cases of Stage I, 14 cases of Stage II and 13 cases of Stage III. For patients with cervical inflammation, the average age was $57.6 \pm 14.5$ years, and there were 8 cases $(32.0 \%)$ of positive HPV infection.

Methods and observation indexes. The positive expression rates of FOXC2, E-cadherin, N-cadherin, VEGF, stromal cell-derived factor-1 (SDF-1), Notch protein and lymphatic vessel endothelial hyaluronan receptor-1 (LYVE-1) and expression level of mRNA in cervical lesion tissues were detected using immunohistochemistry and RT-PCR. Data were collected, entered and analyzed by a third party.

Immunohistochemical staining. The conventional tissue sections were made with the thickness of $5 \mu \mathrm{m}$, followed by xylene dewaxing, gradient ethanol hydration and antigen retrieval. Then, a total of $3 \% \mathrm{H}_{2} \mathrm{O}_{2}$ solution was added and the tissue sections were incubated at $27^{\circ} \mathrm{C}$ for $20 \mathrm{~min}$, and normal goat serum working solution was added and incubated at $27^{\circ} \mathrm{C}$ for $30 \mathrm{~min}$. Thereafter, mouse monoclonal FOXC2 antibody (dilution, 1:500; cat. no. ab55004), mouse monoclonal E-cadherin antibody (dilution, 1:500; cat. no. ab1416); mouse monoclonal N-cadherin antibody (dilution, 1:500; cat. no. ab98952); mouse monoclonal VEGF antibody (dilution, 1:500; cat. no. ab69479); mouse monoclonal SDF-1 antibody (dilution, 1:500; cat. no. ab89321); mouse monoclonal Notch antibody (dilution, 1:500; cat. no. ab44986) and mouse
monoclonalLYVE-1 antibody (dilution, 1:500; cat.no. ab33477) were added and incubated in a humid box at $4{ }^{\circ} \mathrm{C}$ overnight. Then goat polyclonal secondary antibody to mouse IgG-H\&L (HRP) (dilution, 1:2,000; Abcam, Cambridge, MA, USA; cat. no. ab6789) was added. The horseradish peroxidase-labeled streptavidin working solution (Sigma-Aldrich, St. Louis, MO, USA) was added and incubated in a humid box at $27^{\circ} \mathrm{C}$ for $20 \mathrm{~min}$, and polybutylene succinate was used to wash the tissue sections for 5 min 3 times, followed by color development using DAB, re-staining using hematoxylin, differentiation using hydrochloric acid alcohol, back to blue using ammonium hydroxide, gradient ethanol dehydration, transparency using xylene, sealing using neutral gum, airing at room temperature and observation under optical microscope (BX-42; Olympus, Tokyo, Japan). The semi-quantitative method was used to determine the results according to the staining intensity and the proportion of stained cells. The cytoplasm or nucleus yellow-stained to dark brown was regarded as positive. No positive staining, 0 point; weak staining, 1 point; moderate staining, 2 points; strong staining, 3 points. Proportion of positive cells $\leq 5 \%, 0$ point; $6-25 \%, 1$ point; $26-50 \%, 2$ point; $51-75 \%, 3$ points; $>75 \%, 4$ points; and the product of both scales: $0-3$ points, negative; $4-12$ points, positive.

PCR method. Total RNA was extracted from cells by conventional Trizol reagent. Concentration and purity were determined by ultraviolet spectrophotometer (Hitachi, Tokyo, Japan). cDNA was produced by reverse transcription kit. The primer sequences were synthesized by Sangon Biotech Co., Ltd. (Shanghai, China) according to the sequence of GenBank (Table I). The reaction system was as follows: $2 \mu \mathrm{l}$ cDNA $+3 \mu \mathrm{l}$ 
Table II. Analysis of immunohistochemical positive rate [case (\%)].

\begin{tabular}{|c|c|c|c|c|c|c|c|}
\hline Group & FOXC2 & E-cadherin & N-cadherin & VEGF & SDF-1 & Notch & LYVE-1 \\
\hline Cervical cancer $(n=66)$ & $45(68.2)$ & $15(22.7)$ & $50(75.8)$ & $41(62.1)$ & $39(59.1)$ & $35(53.0)$ & $49(74.2)$ \\
\hline CIN $(n=42)$ & $20(47.6)$ & $22(52.4)$ & $18(42.9)$ & $15(35.7)$ & $13(31.0)$ & $10(23.8)$ & $16(38.1)$ \\
\hline Inflammation $(n=25)$ & $7(28.0)$ & $20(80.0)$ & $3(12.0)$ & $4(16.0)$ & $2(8.0)$ & $3(12.0)$ & $4(16.0)$ \\
\hline
\end{tabular}

FOXC2, forkhead box C2; VEGF, vascular endothelial growth factor; SDF-1, stromal cell-derived factor-1; LYVE-1, lymphatic vessel endothelial hyaluronan receptor-1; CIN, cervical intraepithelial neoplasia.

Table III. Analysis of PCR results.

\begin{tabular}{lccccccc}
\hline Group & FOXC2 & E-cadherin & N-cadherin & VEGF & SDF-1 & Notch & LYVE-1 \\
\hline $\begin{array}{l}\text { Cervical } \\
\text { cancer }\end{array}$ & $0.5236 \pm 0.1326$ & $0.1242 \pm 0.0548$ & $0.3452 \pm 0.1235$ & $0.4578 \pm 0.1526$ & $0.3659 \pm 0.1235$ & $0.3547 \pm 0.1635$ & $0.4265 \pm 0.1527$ \\
CIN & $0.3121 \pm 0.1127$ & $0.3568 \pm 0.1214$ & $0.1212 \pm 0.0865$ & $0.2462 \pm 0.1032$ & $0.1023 \pm 0.0656$ & $0.1234 \pm 0.0548$ & $0.2132 \pm 0.1326$ \\
Inflammation & $0.1024 \pm 0.0659$ & $0.6457 \pm 0.2365$ & $0.0654 \pm 0.0123$ & $0.1123 \pm 0.0865$ & $0.0547 \pm 0.0212$ & $0.0659 \pm 0.0126$ & $0.1027 \pm 0.0659$
\end{tabular}

FOXC2, forkhead box C2; VEGF, vascular endothelial growth factor; SDF-1, stromal cell-derived factor-1; LYVE-1, lymphatic vessel endothelial hyaluronan receptor-1; CIN, cervical intraepithelial neoplasia.

upstream primer and $3 \mu 1$ downstream primer $+0.5 \mu 1$ Taq polymerase $+1 \mu \mathrm{l}$ dNTPs $+3 \mu \mathrm{l} \mathrm{MgCl}_{2}+5 \mu \mathrm{l}$

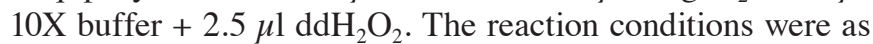
follows: $95^{\circ} \mathrm{C}$ for $5 \mathrm{~min}, 95^{\circ} \mathrm{C}$ for $30 \mathrm{sec}, 58^{\circ} \mathrm{C}$ for $30 \mathrm{sec}, 72^{\circ} \mathrm{C}$ for $60 \mathrm{sec}$, a total of 30 cycles, $72^{\circ} \mathrm{C}$ for $10 \mathrm{~min}$. PCR products were identified using $2 \%$ agarose gel electrophoresis, followed by UV spectroscopic imaging using gel image analysis system, and gray value analysis using digital photograph. The results obtained are presented using $2^{-\Delta \Delta \mathrm{Cq}}$ method.

Statistical analysis. Statistical analysis was carried out using SPSS 20.0 software (IBM SPSS, Armonk, NY, USA). Measurement data are presented as mean \pm standard deviation. One-way analysis of variance was used for intergroup comparison, and least significant difference t-test was used for pairwise comparison. Enumeration data are presented as case or percentage (\%). Chi-square test was used for intergroup comparison; Pearson or Chi-square test was used for correlation analysis. $\mathrm{P}<0.05$ indicates that the difference was statistically significant.

\section{Results}

Analysis of immunohistochemical results. The positive expression rates of FOXC2, N-cadherin, VEGF, SDF-1, Notch and LYVE-1 in cervical cancer tissues were significantly higher than those in CIN, and those in the inflammatory tissues were the lowest. The positive expression rate of E-cadherin in cervical cancer tissues was lower than that in CIN, and that in the inflammatory tissues was the highest $(\mathrm{P}<0.05$; Table II).

Analysis of PCR results. The mRNA levels in FOXC2, N-cadherin, VEGF, SDF-1, Notch and LYVE-1 in cervical cancer tissues were significantly higher than those in CIN, and those in the inflammatory tissues were the lowest. The mRNA level in E-cadherin in cervical cancer tissues was lower than that in CIN, and that in the inflammatory tissues was the highest $(\mathrm{P}<0.05$; Table III).

Analysis of clinicalfeatures of tumors. The positive expression rates of FOXC2, E-cadherin, N-cadherin, VEGF, SDF-1, Notch and LYVE-1 in patients with cervical cancer were not related to age, but were related to HPV infection, pathological pattern, clinical stage, maximum tumor diameter and differentiation grade. The positive expression rates of FOXC2, N-cadherin, VEGF, SDF-1, Notch and LYVE-1 in patients with cervical cancer (HPV positive, squamous cell carcinoma, Stages III-IV, maximal diameter $\geq 3.8 \mathrm{~cm}$ and low differentiation) were increased, and the positive expression rate of E-cadherin was decreased $(\mathrm{P}<0.05$; Table IV).

Correlation analysis. Correlation analysis of the immunohistochemical results of patients with cervical cancer revealed that FOXC2 was positively correlated with the positive expression rates of N-cadherin, VEGF, SDF-1, Notch and LYVE-1 $(\mathrm{r}=0.512, \mathrm{P}=0.007 ; \mathrm{r}=0.463, \mathrm{P}=0.013 ; \mathrm{r}=0.412, \mathrm{P}=0.018$; $\mathrm{r}=0.365, \mathrm{P}=0.022 ; \mathrm{r}=0.385, \mathrm{P}=0.019)$, and negatively correlated with E-cadherin $(\mathrm{r}=-0.446, \mathrm{P}=0.011)$.

\section{Discussion}

Seventeen subtribes of forkhead box protein, also known as winged helix protein, have been found, such as A, C, L, O, M, P (10). FOXC2 gene, also known as Mfhl gene, has $94 \%$ homology in human and mouse, which is located on human chromosome 16q22-24 (10). The present findings showed that the abnormal expression of FOXC2, E-cadherin, N-cadherin, 
Table IV. Analysis of clinical features of the tumors [case $(\%)]$.

\begin{tabular}{|c|c|c|c|c|c|c|c|}
\hline Characteristics & $\begin{array}{c}\text { FOXC2 } \\
(n=45)\end{array}$ & $\begin{array}{l}\text { E-cadherin } \\
\quad(n=15)\end{array}$ & $\begin{array}{l}\text { N-cadherin } \\
\quad(n=50)\end{array}$ & $\begin{array}{l}\text { VEGF } \\
(n=41)\end{array}$ & $\begin{array}{l}\text { SDF-1 } \\
(n=39)\end{array}$ & $\begin{array}{l}\text { Notch } \\
(n=35)\end{array}$ & $\begin{array}{c}\text { LYVE-1 } \\
(n=49)\end{array}$ \\
\hline \multicolumn{8}{|l|}{ Age, years } \\
\hline$<62(\mathrm{n}=30)$ & 22 & 6 & 22 & 23 & 16 & 15 & 23 \\
\hline$\geq 62(n=36)$ & 23 & 9 & 28 & 18 & 23 & 20 & 26 \\
\hline \multicolumn{8}{|l|}{ HPV } \\
\hline Positive $(n=35)$ & 30 & 3 & 32 & 26 & 27 & 25 & 35 \\
\hline Negative $(n=31)$ & 15 & 12 & 18 & 15 & 12 & 10 & 14 \\
\hline \multicolumn{8}{|l|}{ Pathological pattern } \\
\hline Squamous carcinoma $(n=57)$ & 43 & 9 & 47 & 38 & 37 & 33 & 45 \\
\hline Adenocarcinoma $(\mathrm{n}=9)$ & 2 & 6 & 3 & 3 & 2 & 2 & 4 \\
\hline \multicolumn{8}{|l|}{ Clinical stage } \\
\hline I-II $(n=30)$ & 13 & 10 & 16 & 11 & 11 & 10 & 16 \\
\hline III-IV (n=36) & 32 & 5 & 34 & 30 & 28 & 25 & 33 \\
\hline \multicolumn{8}{|l|}{ Maximum tumor diameter } \\
\hline$<3.8 \mathrm{~cm}(\mathrm{n}=39)$ & 20 & 13 & 26 & 18 & 17 & 15 & 24 \\
\hline$\geq 3.8 \mathrm{~cm}(\mathrm{n}=27)$ & 25 & 2 & 24 & 23 & 22 & 20 & 25 \\
\hline \multicolumn{8}{|l|}{ Differentiation grade } \\
\hline Low $(n=15)$ & 13 & 2 & 14 & 13 & 12 & 11 & 14 \\
\hline Middle and high $(n=51)$ & 32 & 13 & 36 & 28 & 27 & 24 & 35 \\
\hline
\end{tabular}

FOXC2, forkhead box C2; VEGF, vascular endothelial growth factor; SDF-1, stromal cell-derived factor-1; LYVE-1, lymphatic vessel endothelial hyaluronan receptor-1; HPV, human papillomavirus.

VEGF, SDF-1, Notch and LYVE-1 were correlated with the occurrence and progression of cervical cancer. The positive expression rates of FOXC2, N-cadherin, VEGF, SDF-1, Notch and LYVE-1 in patients with cervical cancer (HPV positive, squamous cell carcinoma, Stages III-IV, maximal diameter $\geq 3.8 \mathrm{~cm}$ and low differentiation) were increased, and the positive expression rate of E-cadherin was decreased. FOXC2 was positively correlated with the positive expression rates of N-cadherin, VEGF, SDF-1, Notch and LYVE-1, and negatively correlated with E-cadherin. Therefore, we believe that the high expression of FOXC2 is correlated with the HPV infection, pathological pattern, clinical stage, tumor diameter and differentiation grade of cervical cancer, which may be involved in the EMT, vascular and matrix formation, Notch signaling pathway and lymphangiogenesis.

EMT is an important mechanism of invasion and metastasis of epithelium-derived malignant tumor cells with the outstanding performance of decreased epithelial cells marked by E-cadherin and increased interstitial cells marked by $\mathrm{N}$-cadherin. FOXC2 can inhibit the expression of E-cadherin in the adhesion part of epithelial cells (10). The knockdown of FOXC2 inhibits the movement and invasion of hepatoma carcinoma cells, reduces the expression of N-cadherin, matrix metalloproteinase-2 (MMP-2) and angiopoietin-2 and disturbs the EMT degree (11). EMT is also associated with the formation of breast cancer stem cells. EMT or expression of EMT transcription factor (such as FOXC2) in mammary epithelial cells obtains the tumor stem cell markers $\left(\mathrm{CD} 44^{\text {high }} / \mathrm{CD} 24^{\text {low }}\right)$, and increases the degree of malignancy (12). Angiogenesis is the material basis for tumor proliferation, differentiation, invasion and metastasis. VEGF is a potent cytokine that can induce angiogenesis (13). Sano et al found that the growth speed of transplant subcutaneous sarcoma in mouse with FOXC2 heterozygous mutation (FOXC2 +/-) and the number of new blood vessels were significantly decreased compared with those with wild-type (14). Furthermore, the expression of VEGF, MMP-2 and platelet-derived growth factor B and other angiogenic factors were decreased. FOXC2 can promote the secretion of SDF-1 and activate the chemokine receptor 4, which is a type of membrane receptor in tumor cells (15). Notch signaling pathway is an important channel involved in the regulation of cell proliferation, differentiation, invasion and metastasis in the body. FOXC2 can activate a number of key targets in the Notch signaling pathway, such as the Notch ligand D114 and the downstream genes Heyl/Hey2 $(16,17)$. FOXC2 also plays an important role in lymphatic development. Indeed, lymph vessels in cervical squamous cell carcinoma are mainly distributed in the epithelial cells below the surface area, and the number of lymph vessels in squamous cell carcinoma is higher than that in normal cervical tissue, especially that in the case of lymph node metastasis (18). LYVE-1 is a kind of new hyaluronic acid receptor, which can be specifically expressed in lymphatic endothelial cells of different tissues as the marker of lymphatic development, and is not co-expressed with vascular endothelial markers, CD34 and vWF $(19,20)$. 
This study analyzed the relationship between FOXC2 and clinical features of cervical cancer, and suggested that FOXC2 participates in various mechanisms of tumorigenesis, providing important new targets for improving the early diagnosis, clinical intervention and prognosis evaluation of disease.

\section{References}

1. Yung KW, Yung TT, Chung CY, Tong GT, Liu Y, Henderson J, Welbeck D and Oseni S: Principles of cancer staging. Asian Pac J Surg Oncol 1: 1-16, 2015.

2. Barillari G, Palladino C, Bacigalupo I, Leone P, Falchi M and Ensoli B: Entrance of the Tat protein of HIV-1 into human uterine cervical carcinoma cells causes upregulation of HPV-E6 expression and a decrease in p53 protein levels. Oncol Lett 12: 2389-2394, 2016.

3. Liu J, Cheng Y, He M and Yao S: Vascular endothelial growth factor $\mathrm{C}$ enhances cervical cancer cell invasiveness via upregulation of galectin-3 protein. Gynecol Endocrinol 30: 461-465, 2014.

4. Bohr Mordhorst L, Ahlin C and Sorbe B: Prognostic impact of the expression of Wnt-signaling proteins in cervical carcinoma FIGO stage I-IV treated with radiotherapy or chemoradiotherapy. Oncotarget 7: 63042-63053, 2016.

5. Cai L, Liu D, Lu S, Xu Y and Wang H: The relationship between gene expression of Forkhead box $\mathrm{C} 2$ and tumor progression in cervical carcinoma. Eur J Gynaecol Oncol 35: 625-630, 2014.

6. Pan J, Yang Q, Shao J, Zhang L, Ma J, Wang Y, Jiang BH, Leng $J$ and Bai $X$ : Cyclooxygenase-2 induced $\beta 1$-integrin expression in NSCLC and promoted cell invasion via the EP1/MAPK/E2F-1/FoxC2 signal pathway. Sci Rep 6: 33823, 2016.

7. Werden SJ, Sphyris N, Sarkar TR, Paranjape AN, LaBaff AM, Taube JH, Hollier BG, Ramirez-Peña EQ, Soundararajan R, den Hollander P, et al: Phosphorylation of serine 367 of FOXC2 by p38 regulates ZEB1 and breast cancer metastasis, without impacting primary tumor growth. Oncogene 35: 5977-5988, 2016.

8. Kume T: The role of FoxC2 transcription factor in tumor angiogenesis. J Oncol 2012: 204593, 2012.

9. Dai J, Wang JY, Yang LL, Xiao Y, Qu ZL, Qin SH and Ruan QR: Correlation of Forkhead Box $\mathrm{C} 2$ with subtypes and invasive ability of invasive breast cancer. J Huazhong Univ Sci Techno Med Sci 34: 896-901, 2014.
10. Li C, Ding H, Tian J, Wu L, Wang Y, Xing Y and Chen M: Forkhead box protein $\mathrm{C} 2$ promotes epithelial-mesenchymal transition, migration and invasion in cisplatin-resistant human ovarian cancer cell line (SKOV3/CDDP). Cell Physiol Biochem 39: 1098-1110, 2016

11. Bai X, Wang J, Guo Y, Pan J, Yang Q, Zhang M, Li H, Zhang L, Ma J, Shi F, et al: Prostaglandin E2 stimulates $\beta 1$-integrin expression in hepatocellular carcinoma through the EP1 receptor/PKC/NF-kB pathway. Sci Rep 4: 6538, 2014.

12. Pietilä M, Vijay GV, Soundararajan R, Yu X, Symmans WF, Sphyris N and Mani SA: FOXC2 regulates the G2/M transition of stem cell-rich breast cancer cells and sensitizes them to PLK1 inhibition. Sci Rep 6: 23070, 2016.

13. You W, Gao H, Fan L, Duan D, Wang C and Wang K: Foxc2 regulates osteogenesis and angiogenesis of bone marrow mesenchymal stem cells. BMC Musculoskelet Disord 14: 199, 2013.

14. Sano H, Leboeuf JP, Novitskiy SV, Seo S, Zaja-Milatovic S, Dikov MM and Kume T: The Foxc2 transcription factor regulates tumor angiogenesis. Biochem Biophys Res Commun 392: 201-206, 2010 .

15. Sekuła M, Miekus K and Majka M: Downregulation of the CXCR4 receptor inhibits cervical carcinoma metastatic behavior in vitro and in vivo. Int J Oncol 44: 1853-1860, 2014.

16. Surendran S, S Ramegowda K, Suresh A, Binil Raj SS, Lakkappa RK, Kamalapurkar G, Radhakrishnan N and Kartha CC: Arterialization and anomalous vein wall remodeling in varicose veins is associated with upregulated FoxC2-D114 pathway. Lab Invest 96: 399-408, 2016.

17. Jang IH, Lu YF, Zhao L, Wenzel PL, Kume T, Datta SM, Arora N, Guiu J, Lagha M, Kim PG, et al: Notch1 acts via Foxc2 to promote definitive hematopoiesis via effects on hemogenic endothelium. Blood 125: 1418-1426, 2015.

18. Mellor RH, Tate N, Stanton AW, Hubert C, Mäkinen T, Smith A, Burnand KG, Jeffery S, Levick JR and Mortimer PS: Mutations in FOXC2 in humans (lymphoedema distichiasis syndrome) cause lymphatic dysfunction on dependency. J Vasc Res 48: 397-407, 2011

19. Sotiropoulou N, Bravou V, Kounelis S, Damaskou V, Papaspirou E and Papadaki H: Tumour expression of lymphangiogenic growth factors but not lymphatic vessel density is implicated in human cervical cancer progression. Pathology 42: 629-636, 2010.

20. Fatima A, Wang Y, Uchida Y, Norden P, Liu T, Culver A, Dietz WH, Culver F, Millay M, Mukouyama YS and Kume T: Foxc1 and Foxc2 deletion causes abnormal lymphangiogenesis and correlates with ERK hyperactivation. J Clin Invest 126 2437-2451, 2016. 